\title{
Observational appearance of inefficient accretion flows and jets in 3D GRMHD simulations: Application to Sagittarius $A^{*}$
}

\author{
Monika Mościbrodzka $^{1}$, Heino Falcke ${ }^{1,2}$, Hotaka Shiokawa ${ }^{3}$, and Charles F. Gammie ${ }^{4,5}$ \\ ${ }^{1}$ Department of Astrophysics/IMAPP, Radboud University Nijmegen, PO Box 9010, 6500 GL Nijmegen, The Netherlands \\ e-mail: m.moscibrodzka@astro.ru.nl \\ 2 ASTRON, Oude Hoogeveensedijk 4, 7991 PD Dwingeloo, The Netherlands \\ 3 Department of Physics \& Astronomy, The Johns Hopkins University 3400 N. Charles Street Baltimore, MD 21218, USA \\ 4 Department of Physics, University of Illinois, 1110 West Green Street, Urbana, IL 61801, USA \\ 5 Astronomy Department, University of Illinois, 1002 West Green Street, Urbana, IL 61801, USA \\ e-mail: m.moscibrodzka@astro.ru.nl
}

Received 9 June 2014 / Accepted 22 August 2014

\section{ABSTRACT}

\begin{abstract}
Context. Radiatively inefficient accretion flows (RIAFs) are believed to power supermassive black holes in the underluminous cores of galaxies. Such black holes are typically accompanied by flat-spectrum radio cores indicating the presence of moderately relativistic jets. One of the best constrained RIAFs is associated with the supermassive black hole in the Galactic center, Sgr A*.

Aims. Since the plasma in RIAFs is only weakly collisional, the dynamics and the radiative properties of these systems are very uncertain. Here we want to study the impact of varying electron temperature on the appearance of accretion flows and jets.

Methods. Using three-dimensional general relativistic magnetohydrodynamics accretion flow simulations, we use ray tracing methods to predict spectra and radio images of RIAFs allowing for different electron heating mechanisms in the in- and outflowing parts of the simulations.

Results. We find that small changes in the electron temperature can result in dramatic differences in the relative dominance of jets and accretion flows. Application to Sgr A* shows that radio spectrum and size of this source can be well reproduced with a model where electrons are more efficiently heated in the jet. The X-ray emission is sensitive to the electron heating mechanism in the jets and disk and therefore X-ray observations put strong constraints on electron temperatures and geometry of the accretion flow and jet. For Sgr A*, the jet model also predicts a significant frequency-dependent core shift which could place independent constraints on the model once measured accurately.

Conclusions. We conclude that more sophisticated models for electron distribution functions are crucial for constraining GRMHD simulations with actual observations. For Sgr A*, the radio appearance may well be dominated by the outflowing plasma. Nonetheless, at the highest radio frequencies, the shadow of the event horizon should still be detectable with future Very Long Baseline Interferometric observations.
\end{abstract}

Key words. accretion, accretion disks - black hole physics - magnetohydrodynamics (MHD) - radiative transfer - Galaxy: center galaxies: jets

\section{Introduction}

Supermassive black holes exist in the centers of many galaxies. Observations suggest that plasma flows into the deep gravitational potential of black holes, thereby releasing enormous amounts of energy in the form of radiation and powerful plasma outflows, or jets, that can reach way beyond the host galaxy. Very long baseline interferometry, such as the Event Horizon Telescope (Doeleman et al. 2009) promises to take the first ever images of the event horizon and the plasma flow in the immediate vicinity of black holes (Falcke et al. 2000). Hence, a theoretical understanding of black hole astrophysics is now crucial and timely.

An approximately four million solar mass object detected in the central subarcsecond of the Milky Way is one of the best supermassive black hole candidates (Genzel et al. 2010; Falcke \& Markoff 2013). Interestingly, the supermassive object coincides with the bright radio/millimeter source (Sgr A*). The radiation is most probably of synchrotron origin and is believed to be emitted by a hot, magnetized plasma interacting with the black hole. In the radio band, the size of the source decreases towards shorter wavelengths (Bower et al. 2004, 2014). At millimeter and shorter wavelengths, the radiation is believed to originate in the immediate vicinity of the black hole.

Millimeter Very Long Baseline Interferometry (VLBI) observations measured the average size of $\mathrm{Sgr} \mathrm{A}^{*}$ to be $F W H M_{\lambda=1.3 \mathrm{~mm}}=37_{-10}^{+16} \mu$ as (Doeleman et al. 2008). This size is comparable to the expected diameter of the black hole "shadow" (Falcke et al. 2000). The detection of the black hole shadow would be the first direct evidence of the black hole in the Galactic center. Accurate measurements will be possible in the very near future with enhanced high frequency VLBI observations involving an increasing number of baselines. The detectability of the black hole shadow at millimeter wavelengths will strongly depend on the source geometry close to the black hole horizon.

At $\lambda>1 \mathrm{~mm}$, the source structure is washed out due to scattering of radio waves by electrons in the Milky Way (Bower et al. 2014). Hence, there is still debate concerning the exact origin of the radio emission. Current theories that describe the sources' electromagnetic spectrum reasonably well may be divided into two subgroups: hot magnetized accretion disks; and compact, magnetized jets (e.g. Narayan et al. 1995; Falcke et al. 1993; Yuan et al. 2002). Recently, general relativistic 
magnetohydrodynamic (GRMHD) black hole accretion flow simulations, which often produce relativistic jets (e.g. McKinney 2006; Noble et al. 2007; Beckwith et al. 2008; Tchekhovskoy et al. 2011, and many others), have become available and allow one to merge the two theories in a natural manner.

In Mościbrodzka \& Falcke (2013), we have presented such a merged GRMHD disk plus jet model that is consistent with spectrum and sizes of Sgr A*. We have demonstrated that jets produced by the GRMHD simulations produce the characteristic flat radio spectrum, observed not only in Sgr A* but also in jets in Active Galactic Nuclei, when the electrons in the jet are thermal with a constant temperature and electrons in the disk have a constant ratio of ion to electron temperature. The electron temperature $T_{\mathrm{e}}$ is not explicitly modeled in GRMHD models and can be treated as a model free parameter. Nonetheless, the adopted prescription for $T_{\mathrm{e}}$ is motivated by different physics in the disk and jet regions. The accretion disk is more turbulent and less magnetized in comparison to the jet. The heating of particles might be stronger in a strongly magnetized plasma (e.g. Quataert 1998; Quataert \& Gruzinov 1999).

The present article is an extention of Mościbrodzka \& Falcke (2013). Here, we calculate spectra and images of the two component (disk-plus-jet) models scaled to the size of the Sgr A* system in three dimensions. Our goal is to predict the appearance of the black hole "shadow" at $\lambda=1.3 \mathrm{~mm}$, and to show that visible jets can be produced by GRMHD simulations, with appropriate assumptions about electron thermodynamics. Here, for the first time, we present millimeter $(\lambda=1.3 \mathrm{~mm})$ and radio $(\lambda=3.5-13 \mathrm{~mm})$ images of the merged model.

The present models are improved in comparison to our earlier study in three respects. 1) We extend the previous axisymmetric models to three dimensions. 2) We investigate spectra and images produced by models with various combinations of jet and disk electron temperatures and for the first time present radio images of the jet produced by the RIAF. 3) We include Compton scattering in the radiative transfer scheme to model the spectral energy distribution (SED) up to $\gamma$-ray energies. As we will show, the electron temperatures are constrained by the observed nearinfrared (NIR) and X-ray fluxes.

The present coupled disk plus jet models are still simplified in two respects, at least. First, we do not yet associate the jet electron temperatures, or e-p coupling in the disk, with any particular physical process. The disk and the jet region are described by free but physically reasonable parameters. In our model, we identify inflow and outflow regions and simply ascribe two different electron heating mechanisms, which creates a somewhat artificially sharp boundary between the two.

Second, we assume that the electrons have a purely thermal distribution. The thermal plasma assumption reduces the degrees of freedom in radiative transfer modeling and simplifies our analysis. Electron acceleration into a nonthermal component is nearly inevitable, however. It is the simplest explanation for the NIR flaring component and could explain the high frequency NIR-X-ray spectrum uncovered by multiwavelength campaigns. We will consider a nonthermal component in a future publication.

The article is organized as follows. In Sect. 2 we describe the dynamical and radiative transfer techniques used to calculate spectra and images of the plasma. We also introduce the definition of the jet and the prescription for electron temperatures in the jet and disk zones. The spectra and images produced by the model as a function of the electron temperature are presented in Sect. 3. We summarize the results in Sect. 4.

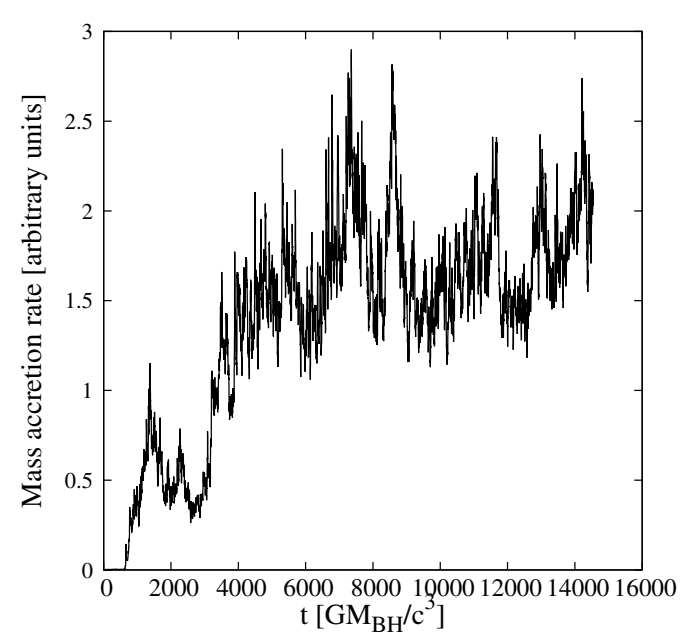

Fig. 1. Evolution of mass accretion rate (in arbitrary units) through the horizon during the entire simulation.

\section{Model of an accretion disk with a jet}

\subsection{Numerical model of accreting black hole}

As time-dependent model of a radiatively inefficient accretion flow onto a black hole we use a three-dimensional (3D) GRMHD simulation carried out by Shiokawa 2013 (run b0-high in Table 5.1 in Shiokawa 2013). The simulation started from a torus in hydrodynamic equilibrium in the equatorial orbit around a rotating black hole (Fishbone \& Moncrief 1976). The torus initially had a pressure maximum at $24 R_{\mathrm{g}}$ and an inner edge at $12 R_{\mathrm{g}}$, where $R_{\mathrm{g}}=G M_{\mathrm{BH}} / c^{2}$. It was seeded with a weak, poloidal field that follows the isodensity contours (single loop model, see Gammie et al. 2003). The dimensionless black hole spin was $a_{*} \simeq 0.94$. The corresponding radius of the event horizon was $r_{\mathrm{h}}=1.348 R_{\mathrm{g}}$ and the inner-most stable circular orbit (ISCO) was located at $r_{\text {ISCO }}=2.044 R_{\mathrm{g}}$. The inner boundary of the computational domain was just inside the event horizon and the outer boundary was at $R_{\text {out }}=240 R_{\mathrm{g}} \simeq 10.7 \mathrm{AU}$, or an angular radius of $\simeq 1.2$ mas (assuming mass and distance of a black hole in the Galactic center to be $M_{\mathrm{BH}}=4.5 \times 10^{6} M_{\odot}$ and $D=8.5 \times 10^{3} \mathrm{PC}$, respectively). The model was evolved for $14000 R_{\mathrm{g}} / c$, which corresponds to $87 \mathrm{~h}$ for the adopted black hole mass (for which the time unit is $R_{\mathrm{g}} / c .=22.17 \mathrm{~s}$ ). This time interval is equivalent to about 19 orbital periods of at $r=24 R_{\mathrm{g}}$.

\subsection{Jet and disk zones}

Figure 1 displays the mass accretion rate evolution over the entire simulation. Early evolution of the magnetized torus is governed by the growth of the magnetorotational instability which leads to the development of turbulence and the accretion of plasma into the central object. At $t \approx 10^{4} G M_{\mathrm{BH}} / c^{3}$, the flow has already reached a quasi-stationary state in which the disk is accreting and well developed jets are present below and above the black hole poles. We analyze a relatively short time sequence of the relaxed portion of the simulation data between $t=10000$ and $t=10800 G M_{\mathrm{BH}} / c^{3}(\sim 5 \mathrm{~h})$.

Following Mościbrodzka \& Falcke (2013), we formally define the jet zones using the Bernoulli parameter $-h u_{\mathrm{t}}$, where $h=\left(\rho_{0}+e+P\right) / \rho_{0}$ is the fluid specific enthalpy and $u_{\mathrm{t}}$ is the covariant time component of the four-velocity. We chose $-h u_{\mathrm{t}}=1.02$ to be a boundary between jet and disk zones. For this value of the Bernoulli parameter the jet narrows to a cone 

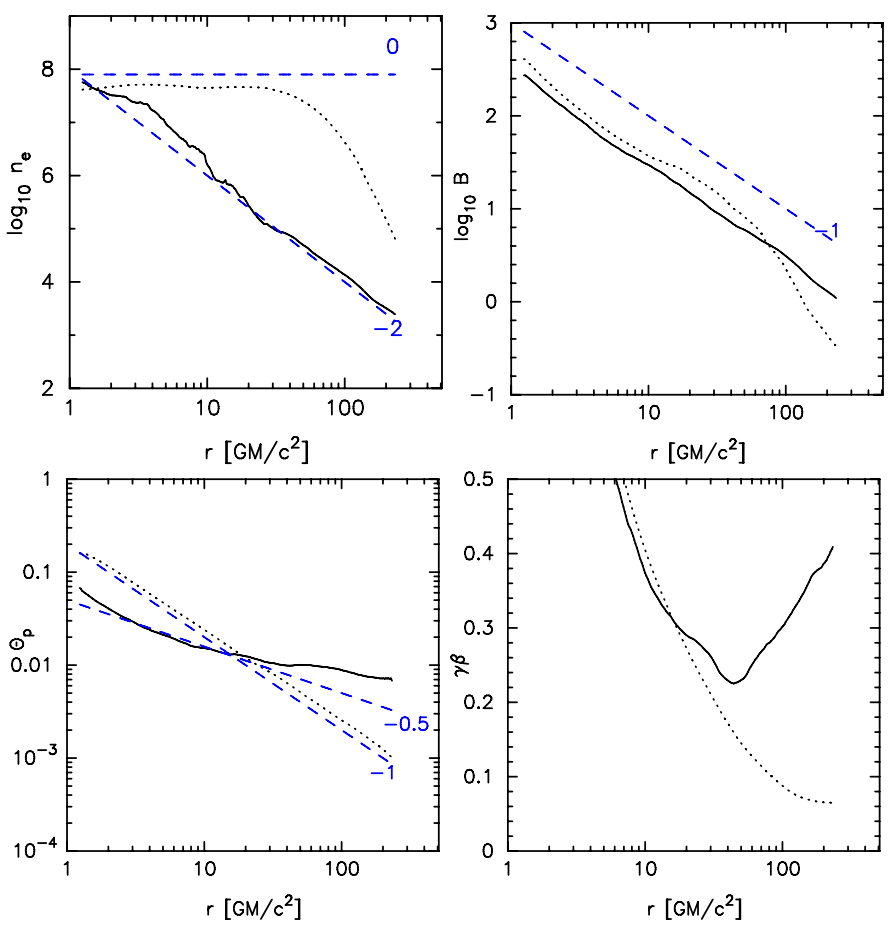

Fig. 2. Shell- and time-averaged profiles of density, magnetic field strength, proton temperature (defined as $\Theta_{\mathrm{p}}=k_{\mathrm{B}} T_{\mathrm{p}} / m_{\mathrm{p}} c^{2}$ ), and plasma speeds measured in the disk and in the jet zones. The time averaging is done over later times during the simulation $(t=$ 10000-10800 GM $M_{\mathrm{BH}} / c^{3}$ ). Solid and dotted lines show the averaged properties in the jet sheath and the disk, respectively. The dashed lines indicate power-law dependencies $r^{p}$, and are labelled with the parameter $p$.

with a half opening angle of approximately $25^{\circ}$ and the definition of the jet excludes the unbound, subrelativistic outflow produced by the outer regions of the disk. Regions with $-h u_{\mathrm{t}}<1.02$ are defined as an accretion flow/wind. One could also adopt another description of jet zones (e.g. Dexter et al. 2012; Sajdowski et al. 2013). We argue that our definition of jet zones is simple and effective and roughly separates outflow and inflow.

Figure 2 shows the radial shell- and time-averaged profiles (computed using Eq. (1) from Mościbrodzka \& Falcke 2013) of density, magnetic field strength, plasma temperature (we plot proton temperature in dimensionless units $\Theta_{\mathrm{p}} \equiv k T_{\mathrm{p}} /\left(m_{\mathrm{p}} c^{2}\right)$ where $T_{\mathrm{p}}$ is computed directly from the simulation) and plasma speed in the jet sheath (i.e. omitting the strongly magnetized but nearly empty jet spine) and in the accretion flow/wind.

In the disk zones, the density and magnetic field follow $r^{0}$ and $r^{-1}$ dependencies, respectively. The radial profile of the density is rather shallow but it is consistent with inflow/outflow RIAF models in which the density profile usually follows a $r^{-3 / 2+s}$ law where $\mathrm{s}$ is a parameter. In time-dependent MHD models parameter $s$ typically ranges from 0.4 to 1 (Begelman 2012; Narayan et al. 2012; Yuan \& Narayan 2014) and one would expect $n_{\mathrm{e}} \sim r^{-0.5}$. In our model, the density has an even shallower profile due to a formal definition of the disk and jet region and due to the fact that our initial disk is rather small from the beginning (initial conditions artifact).

In the jet zones, the density and magnetic field radial distribution roughly follow $r^{-2}$ and $r^{-1}$ dependencies, respectively. The magnetic field strength radial dependence in the $3 \mathrm{D}$ model (but not in the 2D models) reproduces the semi-analytical models of jets needed to explain flat-spectrum radio cored (e.g. de Bruyn 1976; Blandford \& Königl 1979; Falcke \& Biermann 1995; Falcke 1996). The plasma outflowing in the jet sheath starts to accelerate at $r \sim 50 R_{\mathrm{g}}$ and reaches speeds of $\gamma \beta \approx 0.4$ (where $\beta$ is speed measured by the normal observer) at the outer boundary of the simulation. The accretion flow temperatures are virial $\left(T_{\mathrm{p}} \sim r^{-1}\right)$ and in the jet $T_{\mathrm{p}} \sim r^{-0.5}$. Similar dependencies were found in our axisymmetric models (Fig. 1 in Mościbrodzka $\&$ Falcke 2013), which proves the robustness of the two component, spine and sheath, jet solution.

\subsection{Model free parameters and comparison of the model to observations}

The radiative properties of the dynamical model are studied by combining the magnetohydrodynamic model with postprocessing radiative transfer computations. Calculations are carried out using the same tools as in Mościbrodzka et al. (2009). The SEDs and images of the plasma are computed using a Monte Carlo code for relativistic radiative transfer (Dolence et al. 2009) and a ray tracing radiative transfer scheme (Noble et al. 2007). The radiative models include synchrotron emission, absorption and inverse-Compton scattering. Synchrotron emissivities and absorptivities along geodesics trajectories are computed assuming that radiating electrons have a purely thermal energy distribution function. Accurate synchrotron emissivity functions for the thermal electrons are taken from Leung et al. (2011). The radiative transfer codes use all variables, except $T_{\mathrm{e}}$, directly from the accretion flow model.

As in Mościbrodzka \& Falcke (2013), the following prescription for $T_{\mathrm{e}}$ is adopted. In the disk zones (defined in Sect. 2.2), the electron temperature is described using a standard assumption that the electron temperature is a fraction of the proton temperature:

$\frac{k_{\mathrm{b}} T_{\mathrm{e}}}{m_{\mathrm{e}} c^{2}}=\left(\frac{k_{\mathrm{b}} T_{\mathrm{p}}}{\mu m_{\mathrm{p}}}\right)\left(\frac{m_{\mathrm{p}}}{m_{\mathrm{e}}}\right) \frac{1}{\left(T_{\mathrm{p}} / T_{\mathrm{e}}\right)_{\text {disk }}}$,

where $T_{\mathrm{p}}$ is taken from the simulation. In the jet zones, we assume a constant electron temperature

$\frac{k_{\mathrm{b}} T_{\mathrm{e}}}{m_{\mathrm{e}} c^{2}}=\Theta_{\mathrm{e}, \mathrm{j}}=$ const.

The disk $\left(\frac{T_{\mathrm{p}}}{T_{\mathrm{e}}}\right)_{\text {disk }}$ (Eq. (1)) and jet $\Theta_{\text {e,jet }}$ (Eq. (2)) are the modelfree parameters. In our model, we identify disk and jet regions and simply ascribe two different electron heating mechanism, which creates an artificially sharp boundary, so the electron temperature does not transition smoothly from one region to another. The fixed model parameters are the black hole mass, and the distance to the source. The mass of the black hole sets the length scale of the system $G M_{\mathrm{BH}} / c^{2}$, and the distance allows one to translate the absolute system luminosity to an observed luminosity and the physical size of the system to the angular size on the sky. We scale the models to the Galactic center supermassive black hole. We adopt $M_{\mathrm{BH}}=4.5 \times 10^{6} M_{\odot}$, and $D=8.5 \times 10^{3}$ PC. We then vary three free model parameters: the constant electron temperature in the jet $\left(\Theta_{e, j e t}\right)$, the electron to proton temperature ratio in the disk $\left(\left(\frac{T_{\mathrm{p}}}{T_{\mathrm{e}}}\right)_{\text {disk }}\right)$, and the inclination of the observer with respect to the black hole spin $(i)$. All models are normalized to produce the $2.4 \mathrm{Jy}$ flux at $\lambda=1.3 \mathrm{~mm}$ (Doeleman et al. 2008) and the normalization is done by changing the mass accretion rate $\dot{M}$ (i.e. multiplying the matter densities by a constant). Notice that in case of $\mathrm{Sgr} \mathrm{A}^{*}$ the range 
of possible $\Theta_{\mathrm{e}, \text { jet }}$ and $\left(\frac{T_{\mathrm{p}}}{T_{\mathrm{e}}}\right)_{\text {disk }}$ is constrained because $\dot{M}$ is constrained by observations of Faraday rotation of the polarized radio emission $\left(2 \times 10^{-9}<\dot{M}<2 \times 10^{-7} M_{\odot} \mathrm{yr}^{-1}\right.$, Bower et al. 2005; Marrone et al. 2007). Finally, when modeling images of the simulations, we usually assume that the position angle of the black hole spin axis projected on the sky plane is $\xi=0^{\circ}$ East of North direction $(\xi=0$ corresponds to the black hole spin axis inclined at $\sim 60^{\circ}$ relative to the Galaxy's rotation axis, Li et al. 2013).

The scheme to scale the model to the real object is almost identical to the one presented in Mościbrodzka et al. (2009) and (Noble et al. 2007) with a few exceptions: the jet electron temperature is now an independent free parameter; we measure the sizes of the model for $\lambda \geq 1.3 \mathrm{~mm}$; we limit our model survey to only one black hole spin (the spin survey in $3 \mathrm{D}$ is computationally expensive and beyond the scope of the present work).

In Sects. 3.1-3.3 we show general dependencies of the model appearance and spectra on the parameters based on a few chosen examples. In Sect. 3.4, we show a model that best describes emission and size of Sgr A*.

\section{Results}

We have studied 45 radiative transfer models with various combinations of $\Theta_{\mathrm{e}, \text { jet }}$ and $\left(\frac{T_{\mathrm{p}}}{T_{\mathrm{e}}}\right)_{\text {disk }}$ observed at inclination angles of $i=30^{\circ}, 60^{\circ}, 90^{\circ}$. All model parameters together with "normalizing" accretion rates are listed in Table 1.

\subsection{Disk temperature $\left(\frac{T_{p}}{T_{e}}\right)_{\text {disk }}$ dependence}

Figure 3 (upper part) shows "infinite"-resolution (i.e. not accounting for interstellar scattering or finite instrumental resolution) images of models with $\left(\left(\frac{T_{\mathrm{p}}}{T_{\mathrm{e}}}\right)_{\text {disk }}, \Theta_{\mathrm{e}, \text { jet }}\right)=(5,20),(15,20)$ and $(25,20)$ at $\lambda=13,7,1.3 \mathrm{~mm}$. All panels display the same single snapshot of the simulation. Figure 3 (lower part) shows spectra emitted by the three models presented here. The mass accretion rate required to renormalize models to produce the same flux $\lambda=1.3 \mathrm{~mm}$ (marked in the lower part of Fig. 3 as a black point) increases by a factor of 15 from model with $\left(\left(\frac{T_{\mathrm{p}}}{T_{\mathrm{e}}}\right)_{\text {disk }}, \Theta_{\mathrm{e}, \mathrm{jet}}\right)=(5,20)$ to $\left(\left(\frac{T_{\mathrm{p}}}{T_{\mathrm{e}}}\right)_{\text {disk }}, \Theta_{\mathrm{e}, \mathrm{jet}}\right)=$ $(25,20)\left(\dot{M}=3.9 \times 10^{-9}-5.7 \times 10^{-8} M_{\odot} \mathrm{yr}^{-1}\right)$.

The appearance of the model strongly depends on the assumed electron temperatures, as expected. In general, in all models the size of the emission region increases with wavelength but the rate of increase with wavelength is somewhat different. In models with $\left(\left(\frac{T_{\mathrm{p}}}{T_{\mathrm{e}}}\right)_{\text {disk }}, \Theta_{\mathrm{e}, \mathrm{jet}}\right)=(5,20)$ the disk is brighter than the jet at radio wavelengths. The synchrotron photosphere at 7 and $13 \mathrm{~mm}$ is compact and symmetric for the edge-on view. On the other hand, in models with $\left(\left(\frac{T_{\mathrm{p}}}{T_{\mathrm{e}}}\right)_{\mathrm{disk}}, \Theta_{\mathrm{e}, \mathrm{jet}}\right)=(25,20)$ the emission at radio wavelengths is primarily produced by the jet plasma. The emission region in this case is patchy. Evidently, the jet photosphere is significantly more extended in comparison to the disk photosphere. The radio spectra emitted by the model in which the jet emission dominates has a nearly flat-toinverted slope $\left(\alpha_{v} \sim 0.3\right.$, where $\left.F_{v} \sim v^{\alpha}\right)$ in accordance with the analytical predictions; the spectral slope produced by the disk photosphere is significantly steeper $\left(\alpha_{v} \sim 1\right)$.

At submillimeter wavelengths, the plasma becomes optically thin and the spectrum of all models undergoes a slope inversion. At $\lambda=1.3 \mathrm{~mm}$, the emission comes from the direct vicinity of the black hole horizon. In the right panels in Fig. 3, the
Table 1. List of radiative transfer model parameters.

\begin{tabular}{|c|c|c|c|c|}
\hline Model \# & $i$ & $\Theta_{e, j e t}$ & $\left(\frac{T_{\mathrm{p}}}{T_{\mathrm{e}}}\right)_{\text {disk }}$ & $\dot{M}\left[M_{\odot} \mathrm{yr}^{-1}\right]$ \\
\hline 1 & & & 5 & $4.0 \times 10^{-9}$ \\
\hline 2 & & & 10 & $1.3 \times 10^{-8}$ \\
\hline 3 & $90^{\circ}$ & 10 & 15 & $3.7 \times 10^{-8}$ \\
\hline 4 & & & 20 & $7.2 \times 10^{-8}$ \\
\hline 5 & & & 25 & $1.0 \times 10^{-7}$ \\
\hline 6 & & & 5 & $3.9 \times 10^{-9}$ \\
\hline 7 & & & 10 & $1.2 \times 10^{-8}$ \\
\hline 8 & $90^{\circ}$ & 20 & 15 & $2.9 \times 10^{-8}$ \\
\hline 9 & & & 20 & $4.6 \times 10^{-8}$ \\
\hline 10 & & & 25 & $5.7 \times 10^{-8}$ \\
\hline 11 & & & 5 & $3.9 \times 10^{-9}$ \\
\hline 12 & & & 10 & $1.1 \times 10^{-8}$ \\
\hline 13 & $90^{\circ}$ & 30 & 15 & $2.4 \times 10^{-8}$ \\
\hline 14 & & & 20 & $3.6 \times 10^{-8}$ \\
\hline 15 & & & 25 & $4.3 \times 10^{-8}$ \\
\hline 16 & & & 5 & $3.9 \times 10^{-9}$ \\
\hline 17 & & & 10 & $1.3 \times 10^{-8}$ \\
\hline 18 & $60^{\circ}$ & 10 & 15 & $3.2 \times 10^{-8}$ \\
\hline 19 & & & 20 & $6.1 \times 10^{-8}$ \\
\hline 20 & & & 25 & $9.0 \times 10^{-8}$ \\
\hline 21 & & & 5 & $4.4 \times 10^{-9}$ \\
\hline 22 & & & 10 & $1.2 \times 10^{-8}$ \\
\hline 23 & $60^{\circ}$ & 20 & 15 & $2.7 \times 10^{-8}$ \\
\hline 24 & & & 20 & $4.2 \times 10^{-8}$ \\
\hline 25 & & & 25 & $5.4 \times 10^{-8}$ \\
\hline 26 & & & 5 & $4.3 \times 10^{-9}$ \\
\hline 27 & & & 10 & $1.2 \times 10^{-8}$ \\
\hline 28 & $60^{\circ}$ & 30 & 15 & $2.4 \times 10^{-8}$ \\
\hline 29 & & & 20 & $3.5 \times 10^{-8}$ \\
\hline 30 & & & 25 & $4.2 \times 10^{-8}$ \\
\hline 31 & & & 5 & $5.6 \times 10^{-9}$ \\
\hline 32 & & & 10 & $1.4 \times 10^{-8}$ \\
\hline 33 & $30^{\circ}$ & 10 & 15 & $1.0 \times 10^{-8}$ \\
\hline 34 & & & 20 & $5.7 \times 10^{-8}$ \\
\hline 35 & & & 25 & $8.4 \times 10^{-8}$ \\
\hline 36 & & & 5 & $5.6 \times 10^{-9}$ \\
\hline 37 & & & 10 & $1.37 \times 10^{-8}$ \\
\hline 38 & $30^{\circ}$ & 20 & 15 & $2.7 \times 10^{-8}$ \\
\hline 39 & & & 20 & $4.1 \times 10^{-8}$ \\
\hline 40 & & & 25 & $5.2 \times 10^{-8}$ \\
\hline 41 & & & 5 & $5.6 \times 10^{-9}$ \\
\hline 42 & & & 10 & $1.3 \times 10^{-8}$ \\
\hline 43 & $30^{\circ}$ & 30 & 15 & $2.4 \times 10^{-8}$ \\
\hline 44 & & & 20 & $3.4 \times 10^{-8}$ \\
\hline 45 & & & 25 & $4.1 \times 10^{-8}$ \\
\hline
\end{tabular}

shadow of the black hole is visible in all models. At this point, the general and special relativistic effects are equally important in shaping the appearance of the emitting region. The geometry of the emitting region strongly depends on the model parameters. In models with $\left(\left(\frac{T_{\mathrm{p}}}{T_{\mathrm{e}}}\right)_{\text {disk }}, \Theta_{\mathrm{e}, \mathrm{jet}}\right)=(5,20)$ the source angular size is smaller than the diameter of the black hole shadow. The emission is dominated by the left side of the disk where the orbiting plasma is approaching the observer and the emission is therefore strongly Doppler boosted. The image of the 

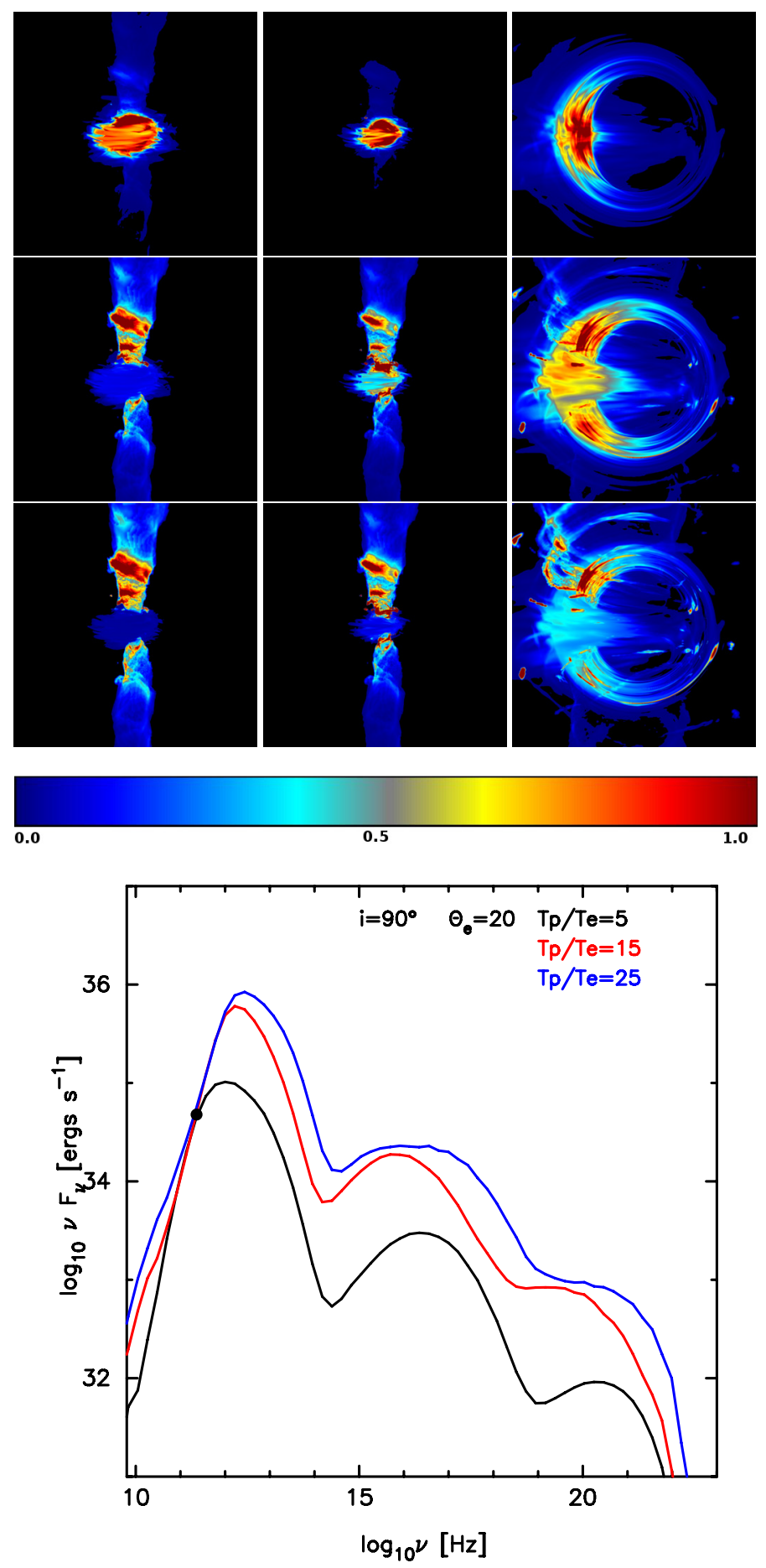

Fig. 3. Dependency on $\left(\frac{T_{\mathrm{p}}}{T_{\mathrm{e}}}\right)_{\text {disk }}$. Upper part: panels from top to bottom display "infinite"-resolution images of models with $\left(\left(\frac{T_{\mathrm{p}}}{T_{\mathrm{e}}}\right)_{\text {disk }}, \Theta_{\text {e,jet }}\right)=$ $(5,20),(15,20)$, and $(25,20)$ at a viewing angle $i=90^{\circ}$. Left, middle, and right columns show the model appearance at $\lambda=13,7$ and $1.3 \mathrm{~mm}$, respectively. Colors code the radiation intensity on a linear scale. The field of view of the left and middle panels is $200 \times 200 \mathrm{GM}_{\mathrm{BH}} / \mathrm{c}^{2}$ (approximately $1 \times 1$ mas), and the right panel's field of view is $20 \times 20 \mathrm{GM}_{\mathrm{BH}} / \mathrm{c}^{2}$. Lower part: spectrum emitted by the various models. The normalization is marked with a black point.

plasma might be described by a Gaussian or a crescent shape and the image morphology is similar to images presented in Noble et al. (2007), Broderick et al. (2009), Dexter et al. (2010), Shcherbakov et al. (2012) and Kamruddin \& Dexter (2013). In models with $\left(\left(\frac{T_{\mathrm{p}}}{T_{\mathrm{e}}}\right)_{\text {disk }}, \Theta_{\mathrm{e}, \mathrm{jet}}\right)=(25,20)$ the black hole shadow becomes more evident because the plasma emission is more extended. In this case the images are also more patchy, similar to emission at $\lambda=7$ and $13 \mathrm{~mm}$. The models are not well represented by a Gaussian or a crescent.

Interestingly, in all models emission at energies higher than NIR is produced primarily via first order inverse-Compton scattering. We find that the scattering occurs mainly in the accretion disk (due to its higher optical depth) within a radial range $r=4-10 G M_{\mathrm{BH}} / c^{2}$. Therefore, the spectrum at high energies strongly depends on the disk electron temperatures, i.e. on $\left(\frac{T_{\mathrm{p}}}{T_{\mathrm{e}}}\right)_{\text {disk }}$. The X-ray to mm flux ratio increases by more than an order of magnitude from models with $\left(\left(\frac{T_{\mathrm{p}}}{T_{\mathrm{e}}}\right)_{\text {disk }}, \Theta_{\mathrm{e}, \mathrm{jet}}\right)=(5,20)$ to $\left(\left(\frac{T_{\mathrm{p}}}{T_{\mathrm{e}}}\right)_{\mathrm{disk}}, \Theta_{\mathrm{e}, \mathrm{jet}}\right)=(25,20)$. These conclusions could change for models that incorporate a nonthermal component in the electron distribution function.

\subsection{Jet temperature $\Theta_{e, j e t}$ dependence}

Figure 4 illustrates the dependency of the model emission on the electron temperature in the jet. In Fig. 4 we show images (at the same wavelengths as in Fig. 3) and spectra of models with $\left(\left(\frac{T_{\mathrm{p}}}{T_{\mathrm{e}}}\right)_{\text {disk }}, \Theta_{\mathrm{e}, \mathrm{jet}}\right)=(20,10),(20,20)$ and $(20,30)$. Here $\dot{M}$ changes by a factor of 2 for models $\left(\left(\frac{T_{\mathrm{p}}}{T_{\mathrm{e}}}\right)_{\text {disk }}, \Theta_{\mathrm{e}, \mathrm{jet}}\right)=(20,10)$ to $\left(\left(\frac{T_{\mathrm{p}}}{T_{\mathrm{e}}}\right)_{\text {disk }}, \Theta_{\mathrm{e}, \mathrm{jet}}\right)=(20,30)$ $\left(\dot{M}=7.2 \times 10^{-8}-3.6 \times 10^{-8} M_{\odot} \mathrm{yr}^{-1}\right)$. While the appearance of the jet at 13 and $7 \mathrm{~mm}$ look similar, there is a dramatic change in the model appearance at $\lambda=1.3 \mathrm{~mm}$. For higher jet temperatures the size of the emitting region decreases. This is because for higher jet temperatures the mass accretion need to be lower (at a fixed $1.3 \mathrm{~mm}$ flux level) and for the fixed $\left(\frac{T_{\mathrm{p}}}{T_{\mathrm{e}}}\right)_{\text {disk }}$ the accretion flow becomes less opaque. Interestingly, the level of NIR emission is sensitive to the jet temperature. Also, the spectral slope in the X-ray band changes from $\Gamma=-0.8$ to $\Gamma=-1.5$ for models with $\Theta_{\mathrm{e}, \mathrm{jet}}=10$ to 30 . The spectral slope change is due to the change of the energy of the seed photons that undergo IC scattering in the disk.

\subsection{Inclination angle i dependence}

In Fig. 5 (same as in Figs. 3 and 4) we show images and spectra depending on $i$. The model shown in the figure has $\left(\left(\frac{T_{\mathrm{p}}}{T_{\mathrm{e}}}\right)_{\text {disk }}, \Theta_{\mathrm{e}, \mathrm{jet}}\right)=(20,20)$, i.e., it possesses a relatively bright jet in comparison to the disk emission. Notice, that the $\dot{M}$ used to normalize the model remains roughly constant for various $i$ $\left(\dot{M}=4.6 \times 10^{-8}-4.1 \times 10^{-8} M_{\odot} \mathrm{yr}^{-1}\right)$.

The emission at $\lambda=13 \mathrm{~mm}$ is predominantly produced by the jet sheath that is much denser in comparison to the jet spine plasma. As a consequence, the jet emission is edge-brightened when viewed at closer to face-on inclination angles. The edge brightening is due to a relativistic Doppler effect. At $i=30^{\circ}$, the effect is observed at both, $\lambda=7$ and $13 \mathrm{~mm}$. At all inclination angles the size of the emission decreases towards shorter wavelengths.

At $\lambda=1.3 \mathrm{~mm}$, the emission comes from the immediate vicinity of the black hole horizon. The images are complex but they show a common feature: the black hole shadow is obvious at any inclination angle. It is more pronounced compared to models 

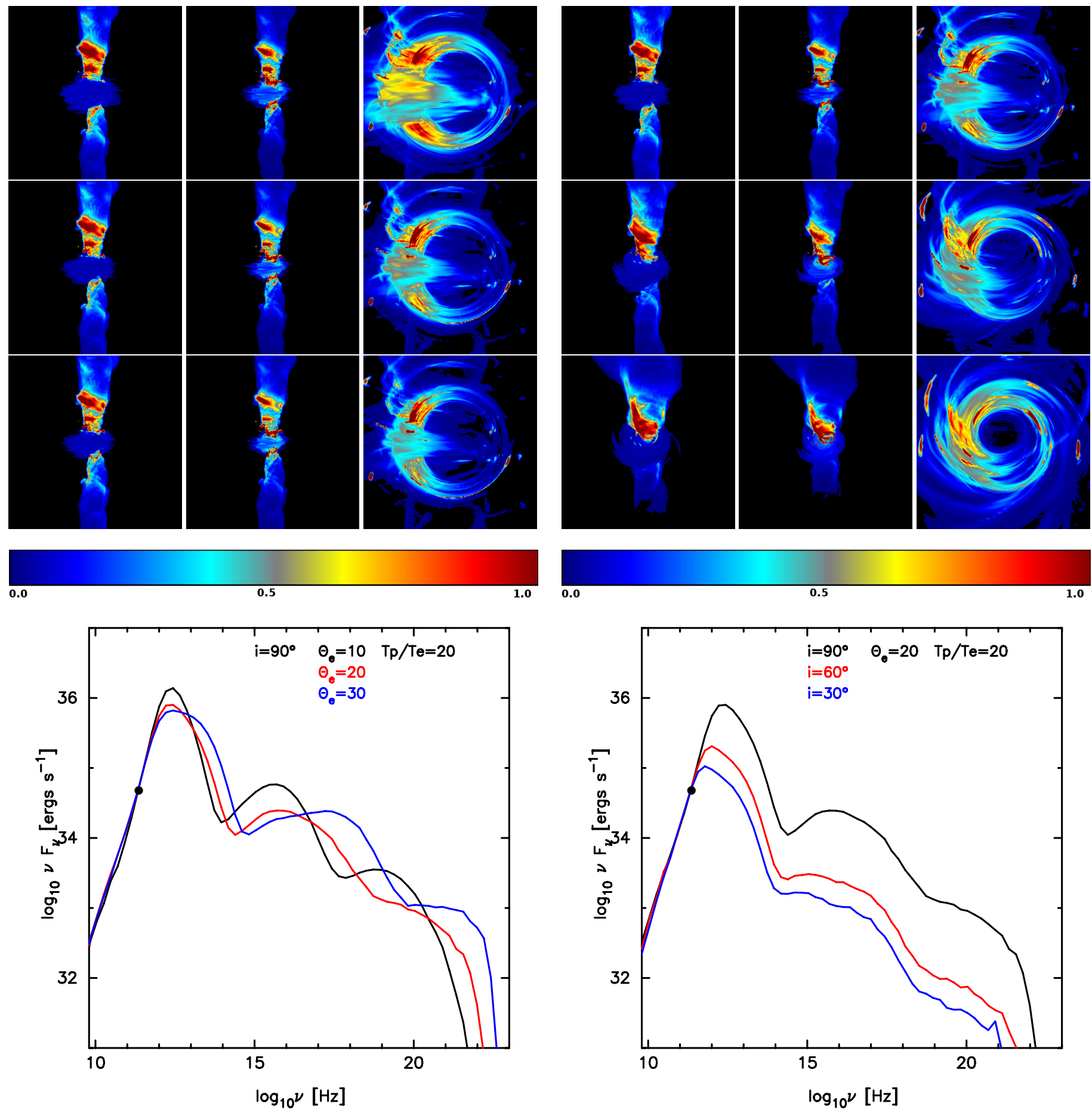

Fig. 4. Same as in Fig. 3, but the dependency on $\Theta_{\text {e,jet }}$ is shown, i.e. images and spectra are for $\left(\left(\frac{T_{\mathrm{p}}}{T_{\mathrm{e}}}\right)_{\text {disk }}, \Theta_{\mathrm{e}, \mathrm{jet}}\right)=(20,10),(20,20)$, and $(20,30)$.

with a bright disk (see e.g. Fig. 3), because the disk emission is more extended, due to a higher $\left(\frac{T_{\mathrm{p}}}{T_{\mathrm{e}}}\right)_{\text {disk }}$ and $\dot{M}$.

The radio SED has a spectral slope $\alpha_{v}=0.3$ and is nearly independent of $i$. This is in contradiction to the analytical finding of Falcke (1996), where the slope was predicted to become flatter with smaller inclination angle. However, this effect is mainly due to differential Doppler beaming and in the present model, the bulk flow in the jet sheath is only very mildly relativistic.

Interestingly, the $\mathrm{X}$-ray to millimeter ratio increases by about 1.5 orders of magnitude for $i$ changing from $30^{\circ}$ to $90^{\circ}$ and hence

Fig. 5. Same as in Fig. 3, but the dependency on the viewing angle $i$ is shown, i.e. images and spectra are for $\left(\left(\frac{T_{\mathrm{p}}}{T_{\mathrm{e}}}\right)_{\text {disk }}, \Theta_{\mathrm{e}, \text { jet }}\right)=(20,20)$ view at $i=90^{\circ}, 60^{\circ}$, and $30^{\circ}$.

could be a potential inclination indicator. The slope of the X-ray emission is constant when varying the inclination angle.

\subsection{Modeling Sgr $A^{*}$}

\subsubsection{Spectra}

We compare SEDs from all 45 models (Table 1) to a selection of the Sgr A* observational data points collected during various observational campaigns (for references to the observations, 


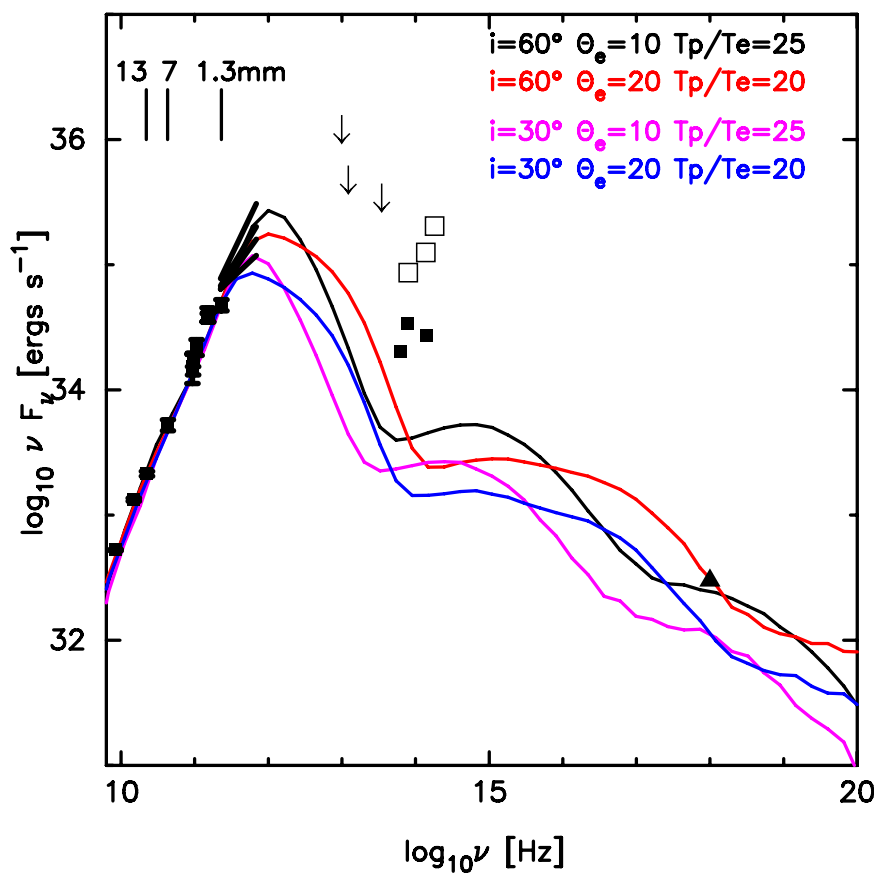

Fig. 6. Full SED (including synchrotron and Compton emission) of models which are roughly consistent with the broadband observations of Sgr A* (models \# 20, 24, 35, and 39, see Table 1.). The observational data points and upper limits are taken from Falcke et al. (1998), An et al. (2005), Marrone et al. (2006), Melia \& Falcke (2001), Doeleman et al. (2008), Schoedel et al. (2011), and the X-ray luminosity of the inner accretion flow is from Neilsen et al. (2013).

see caption of Fig. 6). We average synthetic SEDs in time over the duration of about 3 hours to mimic the quiescent emission of Sgr A*. A model should reproduce the nearly flat radio spectrum $\left(\alpha_{v} \approx 0.3\right.$, Falcke et al. 1998). Recent Chandra observations indicate that the inner accretion flow should contribute at a level of $\sim 10 \%$ to the total quiescent X-ray luminosity, i.e. near the black hole the accretion flow X-ray luminosity should not exceed $L_{X} \approx 4 \times 10^{32} \mathrm{erg} \mathrm{s}^{-1}$ (Neilsen et al. 2013). As shown above, the X-ray emission produced by models strongly depends on the model free parameters. The low X-ray luminosity should in principle easily rule out some of the models.

Indeed, we have found that models with $i=90^{\circ}$ and/or relatively high jet temperatures $\left(\Theta_{\mathrm{e}, \text { jet }} \geq 30\right)$ are too bright in the $\mathrm{X}$-ray band. All models with $\left(\frac{T_{\mathrm{p}}}{T_{\mathrm{e}}}\right)_{\text {disk }}=5-15$ show steep radio spectral slopes $\left(\alpha_{v} \sim 1\right)$ independently of $i$ and $\Theta_{\mathrm{e} \text {,jet }}$ and they too are ruled out from further considerations. There are four models where the spectra are roughly consistent with all observed data points between $\lambda=13 \mathrm{~mm}$ and X-rays (excluding NIR energies at which the source is constantly flaring). These models have jets with $\Theta_{\mathrm{e}, \mathrm{jet}}=10-20$ and relatively cooler disks with $\left(\frac{T_{\mathrm{p}}}{T_{\mathrm{e}}}\right)_{\text {disk }}=20-25$, (models \#20, 24, 35 and 39). Figure 6 displays time-averaged spectra produced by the four models overplotted with the observations of Sgr A*. Models that underpredict the observed variable component of the X-ray luminosity produced by inner accretion flow (\#20,35 and 39) are consistent with the data because they could be made to fit the NIR and X-ray fluxes with the addition of a small non-thermal power-law component in the electron distribution function. The X-ray spectral slope of the model SED is between $\Gamma=-1.8$ (model \#24) and $\Gamma=-1.4$ (model \#20). The mass accretion rates for the four chosen models are in the range between $\dot{M} \approx 4 \times 10^{-8}-9 \times 10^{-8} M_{\odot} \mathrm{yr}^{-1}$, and

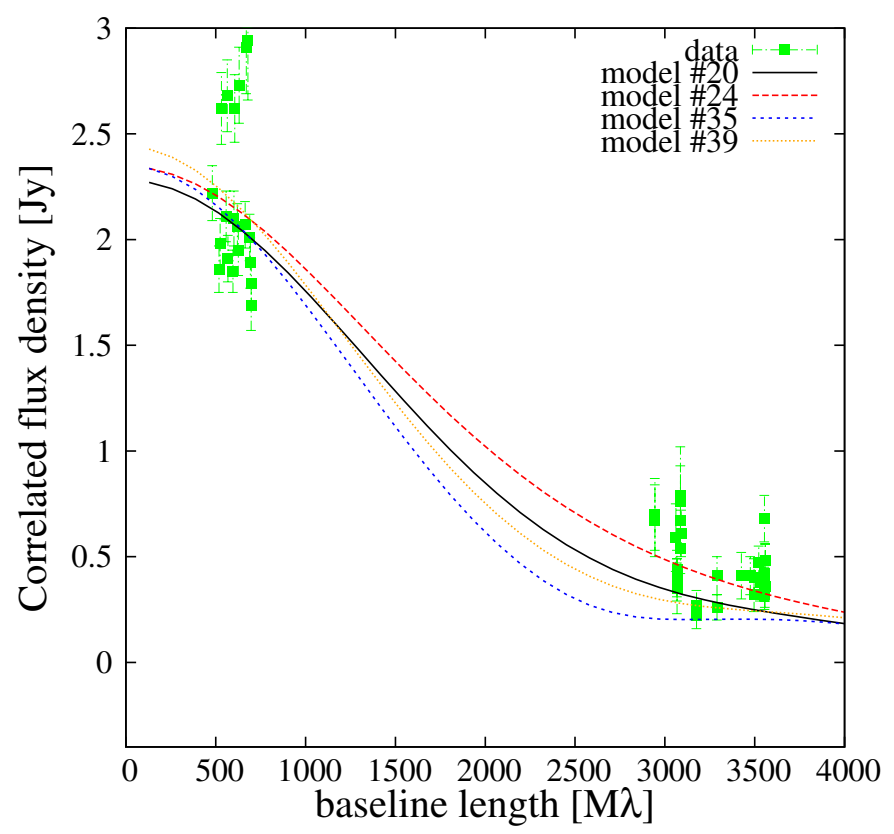

Fig. 7. Visibility profiles for models where the SEDs are shown in Fig. 6 (i.e. models \# 20, 24, 35, and 39). Theoretical visibility amplitudes are computed along the E-W baselines which is roughly the the direction of baselines for which the $1.3 \mathrm{~mm}$-VLBI measurements has been done. The visibility data points from three days of observations are from Fish et al. (2011).

they are fully consistent with the model-dependent $\dot{M}$ imposed by the radio source polarization observations (Bower et al. 2005; Marrone et al. 2007).

\subsubsection{Sizes}

We have studied in greater detail images of the four models with SEDs that are roughly consistent with observations, i.e. models \#20, 24, 35 and 39. We study sizes of images that are timeaveraged over $3 \mathrm{~h}$ similar to the SEDs shown in Fig. 6. Sgr A* has size estimates for $\lambda=1.3 \mathrm{~mm}$ (Doeleman et al. 2008) and for $\lambda=3.5 \mathrm{~mm}-6 \mathrm{~cm}$ (Bower et al. 2006; Shen et al. 2005). At these wavelengths the source angular broadening by the interstellar electron scattering cannot be neglected. To simulate the angular broadening we convolve the intrinsic intensity maps with an elliptical Gaussian function parameterized by

$\begin{aligned} F W H M_{\text {maj }} & =1.309\left(\frac{\lambda^{2}}{1 \mathrm{~cm}}\right) \text { mas } \\ F W H M_{\text {min }} & =0.64\left(\frac{\lambda^{2}}{1 \mathrm{~cm}}\right) \text { mas. }\end{aligned}$

The position angle of the major axis of the scattering Gaussian is $\xi=78^{\circ}$ measured $\mathrm{E}$ of $\mathrm{N}$ on the sky and the FWHMs are taken from long wavelengths observations by Bower et al. (2004) and Bower et al. (2006). We compare the model sizes to observational data at $\lambda=1.3,3.5,7$ and $13 \mathrm{~mm}$. The radiation at $\lambda \geq 13 \mathrm{~mm}$ cannot be modeled in our set-up because it is emitted beyond the adopted outer boundary of our model. Overall, we find that some orientations are consistent with the measured size of Sgr A*.

First, we check if the size of the emitting region at $\lambda=1.3 \mathrm{~mm}$ is consistent with the $\mathrm{mm}$-VLBI measurements. Doeleman et al. (2008) measured and fit the observed Sgr A* 

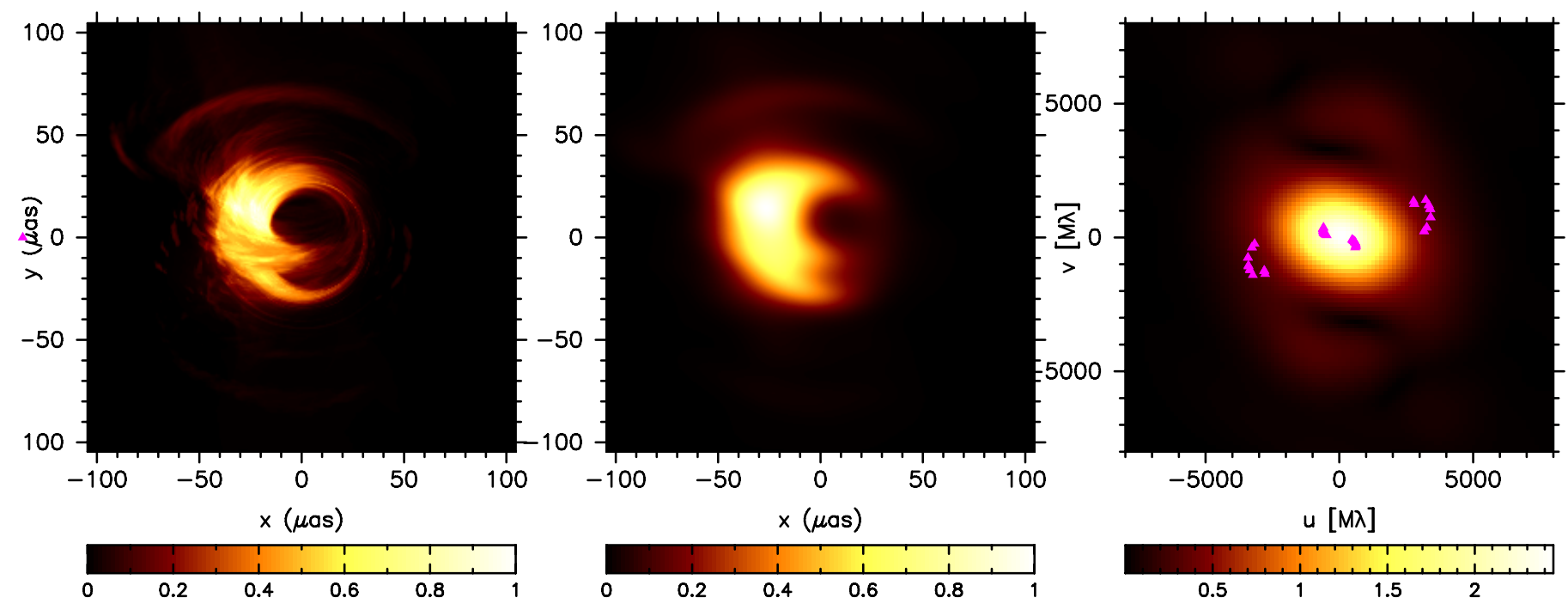

Fig. 8. Intrinsic image, scatter-broadened image, and visibility amplitude distribution for model 24 at $\lambda=1.3 \mathrm{~mm}$. Images are time-averaged (over $\Delta t \approx 3 \mathrm{~h}$ ) and the color intensity indicates the intensity of radiation normalized to unity (linear scale). The visibility amplitudes are in units of Jansky. The visibility $u-v$ tracks are from Fish et al. (2011).

visibilities with a symmetric Gaussian function which correspond to the size of the source of $F W H M_{\text {symm.Gaussian }}=$ $43_{-8}^{+14} \mu$ as (size estimate including angular broadening by scattering with a $F W H M=22 \mu$ as symmetric Gaussian). Our scattering Gaussian function and the source models are strongly asymmetric. We therefore turn to a direct comparison of the model to the visibility data obtained by $1.3 \mathrm{~mm}$-VLBI observations. In particular, we use data obtained by Fish et al. (2011). We compute the theoretical visibility amplitudes (Fourier transformations) of the time-averaged, scatter-broadened model images. Figure 7 shows model 20, 24, 35, and 39 visibility profiles along the E-W direction which roughly corresponds to the Doeleman et al. (2008) and Fish et al. (2011) orientation of the VLBI baselines. Overall, the models with $i=60^{\circ}$ tend to show better alignment with the observed data points in comparison to models with $i=30^{\circ}$ which seem to be too extended to fit the data (because $\left.F W H M_{\mathrm{uv}} \sim 1 / F W H M_{x y}\right)$.

The best fit model is \#24. Figure 8 shows the model image, scatter-broadened image, and visibility amplitude for the black hole spin position angle $\xi=0^{\circ}$ with respect to the N-E direction. The visibility amplitudes are overplotted with the mm-VLBI u-v tracks from Fish et al. (2011). The time-averaged image of the model is composed of a ring, crescent-like shape, and a spotlike emission which constitutes the image centroid. The ringlike shape is produced by a gravitationally lensed image of the accretion flow and the crescent-like "tongue" is an emission feature produced by the approaching side of the disk, which sweeps across the near side of the black hole. The spot-like emission is produced by the foot-point of the isothermal jet. The structure is smeared by the scattering effects, but the shadow of the black hole horizon is clearly visible. The black hole shadow is detectable in the visibility space as two minima located at baselines nearly orthogonal to the currently available ones.

What does model \#24 look like at $\lambda>1.3 \mathrm{~mm}$ ? Figure 9 displays time-averaged (over $\Delta t=3 \mathrm{~h}$, same as $1.3 \mathrm{~mm}$ images) images of the model \#24 at $\lambda=3.5,7$ and $13 \mathrm{~mm}$ and a $\xi=0^{\circ}$. The left panels are the intrinsic emission maps and the rightmost panels show the images after convolution with the asymmetric scattering Gaussian (Eqs. (3)). In the right panel in Fig. 9 , at $\lambda=3.5 \mathrm{~mm}$ a weak jet feature is visible. Interestingly, in this model the shadow of the black hole is already visible in the non-scattered images at this frequency. At $\lambda=7 \mathrm{~mm}$ and $13 \mathrm{~mm}$, the source looks, as expected, Gaussian-like despite the underlying jet-structures.

For $\lambda=3.5,7$ and $13 \mathrm{~mm}$, we measure the size of the emitting region by fitting an asymmetric Gaussian function to the modeled scatter-broadened images (as those in Fig. 9). Figure 10 shows the radio source sizes (major FWHM) at $\lambda=0.1-13 \mathrm{~mm}$. The size-wavelength relation might be altered when non-thermal particle tails are added to the distribution functions (e.g. Özel et al. 2000). At $\lambda=1.3 \mathrm{~mm}$ the data point is produced by the symmetric Gaussian fit to the visibility data so the discrepancy between the model and the data can be ignored. At $\lambda>13 \mathrm{~mm}$, the model and the source follows the $\lambda^{2}$-law expected from scattering. For intermediate wavelengths $(\lambda=3.5,7$, and $13 \mathrm{~mm})$ the sizes of the model major axis are following the same dependency as the data points but also depend on the chosen position angle of the jet axis (or black hole spin) on the sky. Not only is the model consistent with the data (given the uncertainties in the electron distribution function) but it is interesting that the size-wavelength relation contains information about the position angle of the jet, and therefore the black hole spin axis, on the sky.

Finally, it is worth mentioning that models with a bright jet, like the one considered in this subsection (model \#24) predict a significant shift of the image centroid as a function of wavelength (so called core-shift). Figure 11 shows the model predictions for the emission centroid position as a function of frequency. For $\lambda>3.5 \mathrm{~mm}$ the core shift is roughly proportional to the wavelength, which is consistent with predictions from the analytical jet models (Falcke \& Biermann 1999). A source shift of about $300 \mu$ as is predicted by the model when moving from 1.3 to $13 \mathrm{~mm}$ observations. Measurements of the core shift would lead to the constraints of the position angle of an eventual jet on the sky and would put an independent constraint on the models.

\section{Summary}

We have shown broadband spectra $(\lambda=13 \mathrm{~mm}-\gamma$-rays $)$ and radio images $(\lambda=13,7,3.5$, and $1.3 \mathrm{~mm})$ of GRMHD radiatively inefficient accretion flow models for various electron temperature prescriptions in inflowing and outflowing plasma of 

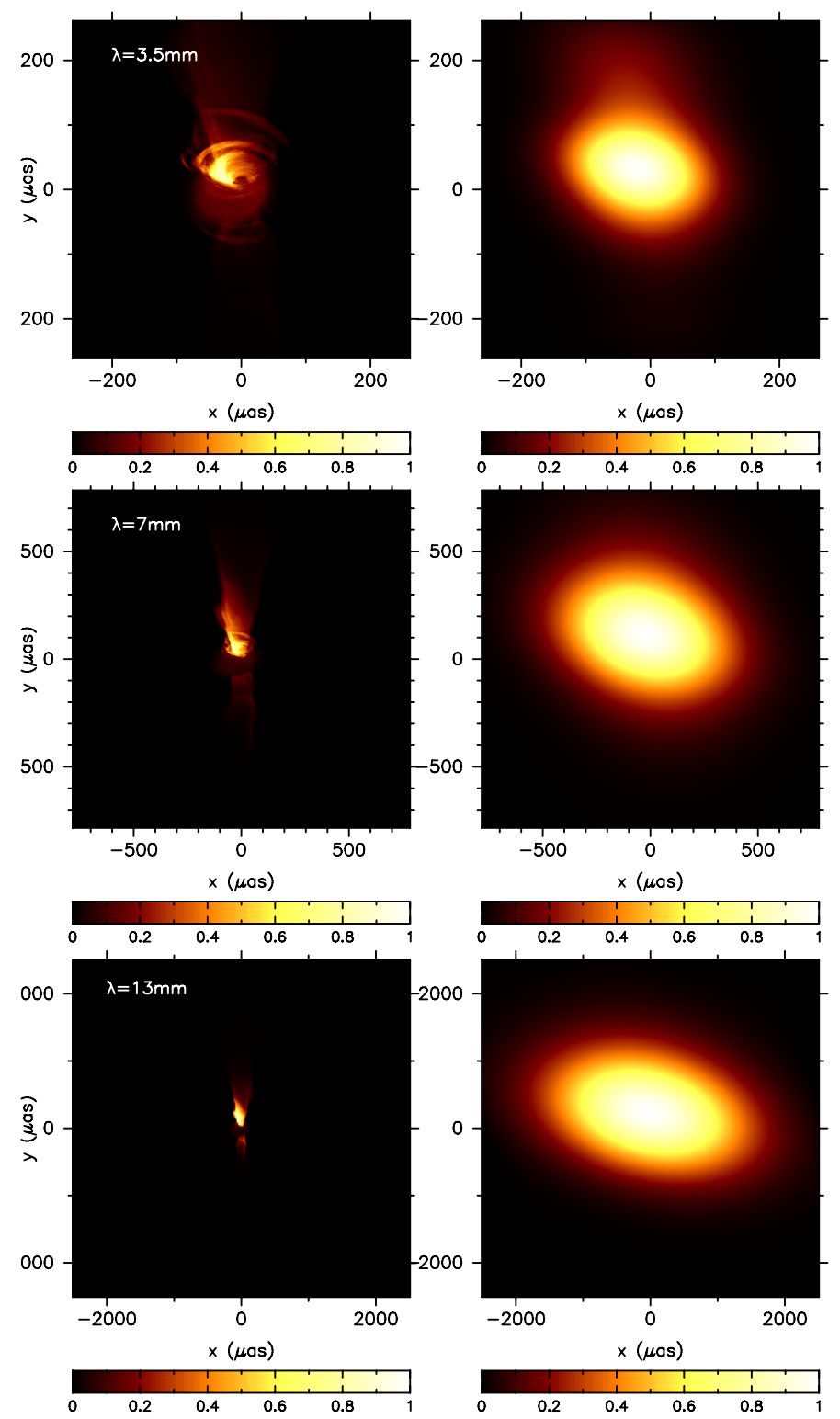

Fig. 9. Time-averaged images of model \#24 at $\lambda=13,7$ and $3.5 \mathrm{~mm}$. Color intensity codes the intensity of radiation normalized to unity (linear scale). Left panel: intrinsic intensity distribution, right panel: intensity distribution convolved with a asymmetric Gaussian function to simulate the effect of angular-broadening by scattering of radio waves on the free electrons along the line of sight.

an underluminous black hole. We have found that the assumed prescription for the electron temperature makes large qualitative difference in the model appearance and emission. The presented results based on the 3D models are broadly consistent with those obtained from 2D models in Mościbrodzka \& Falcke (2013).

It is possible to consider more sophisticated models for the electrons temperatures by, for instance, assuming different electron-proton coupling constants in the disk and in the jet with a natural transition controlled by, e.g., the magnetization of the plasma. Even more realistic models should include evolving the electron energy equation self-consistently with the plasma dynamics. Future RIAF models should also include, for example, the effects of pressure anisotropy and thermal conduction, which may naturally lead to different electron distribution functions in the turbulent accretion flow and in the jet.

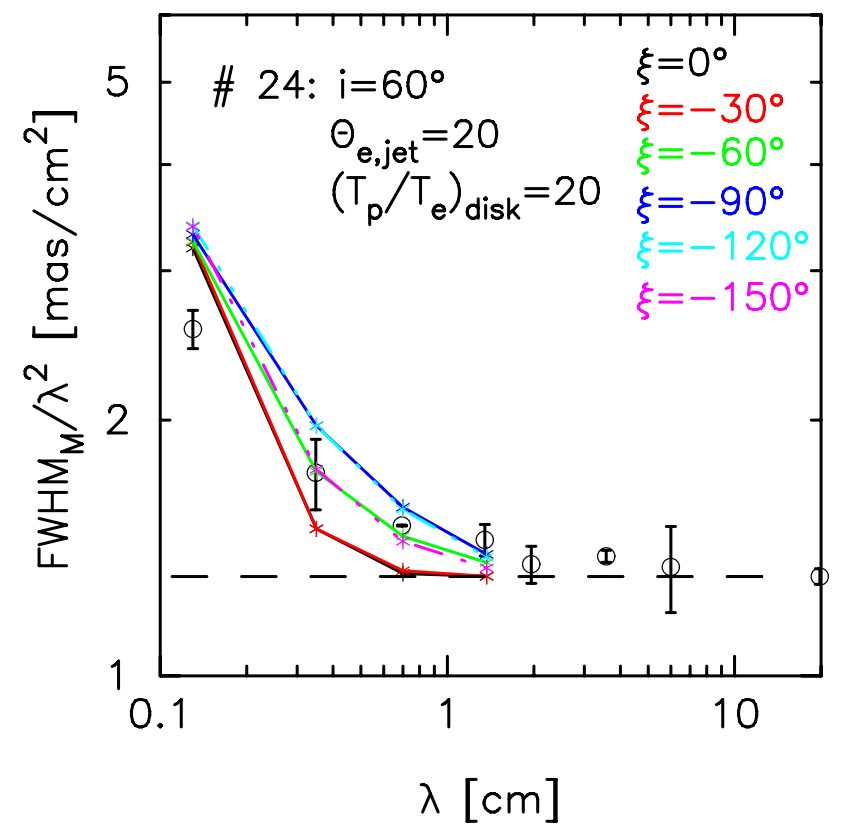

Fig. 10. Wavelength-size relation for model 24 oriented at various position angles with respect to the NE direction. The colored lines correspond to various position angles of the black hole spin on the sky, $\xi$. Size for $\lambda>13 \mathrm{~mm}$ are not modeled because they fall outside our simulation domain. All observational data taken from Bower et al., except one point at $1.3 \mathrm{~mm}$ which is taken from Doeleman et al. (2008), which is a fit to the symmetric Gaussian function (due to a poor uv coverage at $1.3 \mathrm{~mm}$ ). At $1.3 \mathrm{~mm}$ the average of major and minor axis of the source are in agreement with the measured size. Horizontal dashed line corresponds to $F W H M_{\text {maj }}=1.309 \lambda^{2}$.

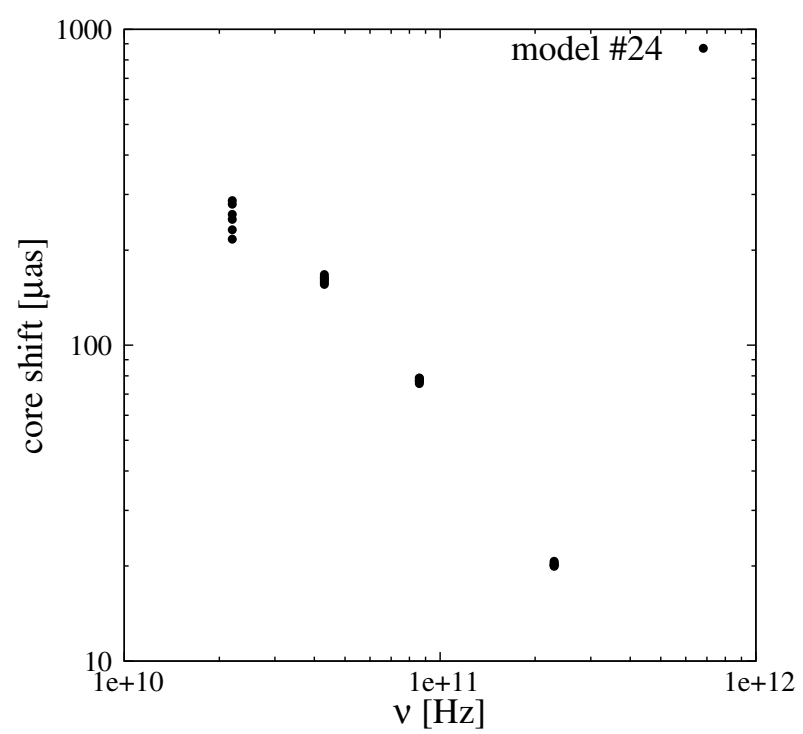

Fig. 11. Position of the model \#24 image centroid as a function of frequency (core-shift).

We have scaled our models to Sgr A* and we can account for most of its observed characteristics: flat radio spectrum, increase of the source size with wavelength and weak X-ray emission. Models where the radio emission is dominated by emission from the jet fit the data best. In particular our present best (bright jet) model, in contrast to the "best-bet" model from Mościbrodzka et al. (2009), produces X-ray luminosity that is consistent with the new X-ray luminosities provided by very long exposure Chandra observations (Neilsen et al. 2013). 
Sgr $A^{*}$ is strongly scatter-broadened at radio wavelengths and hence the orientation of the system and detailed structure cannot be derived directly from VLBI observations yet. Fortunately, the scattering decreases with $\lambda^{2}$-law and at millimeter wavelengths the source structure can be directly observed with a millimeter-VLBI network. Nonetheless, current $\mathrm{mm}$-VLBI and X-ray observations already rule out a large fraction of the parameter range for models.

Eventually, improved mm-VLBI and flux monitoring observations will constrain the geometry of the collisionless plasma in the immediate vicinity of the black hole better. Thus, the ever more sophisticated RIAF models will be constrained more and more tightly in the future, providing us with an excellent opportunity to understand accretion and outflow in low-power supermassive black holes as well as test general relativity reliably with observations of the black hole shadow.

Acknowledgements. This work is supported by NWO Spinoza Price awarded to Heino Falcke and by ERC Synergy Grant "BlackHoleCam: Imaging the Event Horizon of Black Holes" awarded to Heino Falcke, Michael Kramer and Luciano Rezzolla. This work was supported in part by US NSF grants AST 07-09246 and AST 13-33612, and NASA grant NNX10AD03G. This work used XSEDE, which is supported by NSF grant ACI-1053575. MM would like to thank Ryuichi Kurosawa for his comments.

\section{References}

Beckwith, K., Hawley, J. F., \& Krolik, J. H. 2008, ApJ, 678, 1180

Begelman, M. C. 2012, MNRAS, 420, 2912

Blandford, R. D., \& Königl, A. 1979, ApJ, 232, 34

Bower, G. C., Falcke, H., Herrnstein, R. M., et al. 2004, Science, 304, 704

Bower, G. C., Falcke, H., Wright, M. C., \& Backer, D. C. 2005, ApJ, 618, L29

Bower, G. C., Goss, W. M., Falcke, H., Backer, D. C., \& Lithwick, Y. 2006, ApJ, 648, L127

Bower, G. C., Markoff, S., Brunthaler, A., et al. 2014, ApJ, 790, 1

Broderick, A. E., Fish, V. L., Doeleman, S. S., \& Loeb, A. 2009, ApJ, 697, 45

de Bruyn, A. G. 1976, A\&A, 52, 439
Dexter, J., Agol, E., Fragile, P. C., \& McKinney, J. C. 2010, ApJ, 717, 1092 Dexter, J., McKinney, J. C., \& Agol, E. 2012, MNRAS, 421, 1517

Doeleman, S. S., Weintroub, J., Rogers, A. E. E., et al. 2008, Nature, 455, 78

Doeleman, S., Agol, E., Backer, D., et al. 2009, Astronomy, 2010, 68

Dolence, J. C., Gammie, C. F., Mościbrodzka, M., \& Leung, P. K. 2009, ApJS, 184,387

Falcke, H. 1996, ApJ, 464, L67

Falcke, H., \& Biermann, P. L. 1995, A\&A, 293, 665

Falcke, H., \& Biermann, P. L. 1999, A\&A, 342, 49

Falcke, H., \& Markoff, S. B. 2013, Class. Quant. Grav., 30, 244003

Falcke, H., Mannheim, K., \& Biermann, P. L. 1993, A\&A, 278, L1

Falcke, H., Goss, W. M., Matsuo, H., et al. 1998, ApJ, 499, 731

Falcke, H., Melia, F., \& Agol, E. 2000, ApJ, 528, L13

Fish, V. L., Doeleman, S. S., Beaudoin, C., et al. 2011, ApJ, 727, L36

Fishbone, L. G., \& Moncrief, V. 1976, ApJ, 207, 962

Genzel, R., Eisenhauer, F., \& Gillessen, S. 2010, Rev. Mod. Phys., 82, 3121

Kamruddin, A. B., \& Dexter, J. 2013, MNRAS, 434, 765

Leung, P. K., Gammie, C. F., \& Noble, S. C. 2011, ApJ, 737, 21

Li, Z., Morris, M. R., \& Baganoff, F. K. 2013, ApJ, 779, 154

Marrone, D. P., Moran, J. M., Zhao, J.-H., \& Rao, R. 2007, ApJ, 654, L57

McKinney, J. C. 2006, MNRAS, 368, 1561

Mościbrodzka, M., \& Falcke, H. 2013, A\&A, 559, L3

Mościbrodzka, M., Gammie, C. F., Dolence, J. C., Shiokawa, H., \& Leung, P. K. 2009, ApJ, 706, 497

Narayan, R., Yi, I., \& Mahadevan, R. 1995, Nature, 374, 623

Narayan, R., SÄ dowski, A., Penna, R. F., \& Kulkarni, A. K. 2012, MNRAS, 426,3241

Neilsen, J., Nowak, M. A., Gammie, C., et al. 2013, ApJ, 774, 42

Noble, S. C., Leung, P. K., Gammie, C. F., \& Book, L. G. 2007, Class. Quant. Grav., 24, 259

Özel, F., Psaltis, D., \& Narayan, R. 2000, ApJ, 541, 234

Quataert, E. 1998, ApJ, 500, 978

Quataert, E., \& Gruzinov, A. 1999, ApJ, 520, 248

Sąowski, A., Narayan, R., Penna, R., \& Zhu, Y. 2013, MNRAS, 436, 3856

Shcherbakov, R. V., Penna, R. F., \& McKinney, J. C. 2012, ApJ, 755, 133

Shen, Z.-Q., Lo, K. Y., Liang, M.-C., Ho, P. T. P., \& Zhao, J.-H. 2005, Nature, 438,62

Tchekhovskoy, A., Narayan, R., \& McKinney, J. C. 2011, MNRAS, 418, L79

Yuan, F., \& Narayan, R. 2014, ARA\&A, in press [arXiv: 1401.0586]

Yuan, F., Markoff, S., \& Falcke, H. 2002, A\&A, 383, 854 